Tôhoku Math. Journ.

21(1969), 328-335.

\title{
SOME REMARKS ON ANALYTIC CONTINUATIONS
}

\author{
Shinji Yamashita \\ (Received January 31, 1969)
}

1. The purpose of the present paper is to prove some theorems concerning continuations of analytic functions across simple open arcs. Here, a simple open arc means a topological image of the open interval $\{t ; 0<t<1\}$.

Let $D_{1}$ and $D_{2}$ be Jordan domains in the $z$-plane having no point in common and $I$ be a simple open arc lying on the non-empty common boundary of $D_{1}$ and $D_{2}$. Then there arises the following

Problem. Given two analytic functions $f_{1}$ and $f_{2}$ in $D_{1}$ and $D_{2}$ respectively, we set $f=f_{1}$ in $D_{1}$ and $f=f_{2}$ in $D_{2}$. Under what condition do there exist an open subset $I^{*}$ of $I$ and an analytic function $F(z)$ in $D_{1} \cup I^{*} \cup D_{2}$ such that $F(z)=f_{j}(z)$ for $z \in D_{j}(j=1,2)$ ? In other words, under what conditions on $f$ and $I$ can $f$ be extended analytically to an open subset $I^{*}$ of $I$ ?

This problem was investigated by some authors, e.g., Carleman [5], Wolf [14], Meier [8] and from cluster-sets-theoretic viewpoint, Bagemihl [3] gave an answer to this problem under the restriction of $I$ being an open interval on a straight line. Recently, Noshiro [9] gave an improvement of Bagemihl's theorem [3] (cf. also [10]).

First in $\$ 2$ we shall prove an analogous theorem to Bagemihl-Noshiro's in the case where $I$ is an open locally rectifiable arc. Instead of the condition (c) in Theorem 6 in [9] we shall give a global restriction to $f$. In $\$ 3$ we assume that $I$ is a simple open smooth arc. We give an answer to the problem under the condition that $f_{j}$ belongs to the Hardy class $H_{p}\left(D_{j}\right)$ for $p>1(j=1,2)$. In $\$ 4$ we assume that $I$ is a simple open analytic arc. Under the weaker condition that $f_{j}$ is in the class $H_{1}\left(D_{j}\right)(j=1,2)$, we shall give another answer to the problem. Finally in $\$ 5$ we shall state some remarks on null-sets for the class $H_{p}, p \geqq 1$, as applications of two theorems in $\S 3$ and in $\S 4$.

2. By an open locally rectifiable arc $I$ we mean a simple open arc such that every point of $I$ has a neighbourhood which is a rectifiable subarc of $I$. 
We remark that a rectifiable simple arc must be a topological image of the closed interval $\{t ; 0 \leqq t \leqq 1\}$. We also remark that a rectifiable arc has a tangent at every point except for a set of linear measure zero. Here a subset $E$ of an open locally rectifiable arc $I$ is said to be of linear measure zero if for any $\varepsilon>0$, there exists a countable number of open subarcs $\left\{I_{n}\right\}$ of $I$ such that $\bigcup I_{n} \supset E$ and $\sum m I_{n}<\varepsilon$, where $m$ denotes the linear measure (the length).

An analytic function $f$ in a plane domain $D$ is said to be in the class $S(D)$ provided that the subharmonic function $\log ^{+}|f|=\max (\log |f|, 0)$ admits a harmonic majorant in $D$ which is quasi-bounded, i.e., the limiting function of a monotone non-decreasing sequence of non-negative bounded harmonic functions in $D$ (cf. e.g., [15]).

An analytic function $f$ in $D$ is said to be in the Hardy class $H_{p}(D)$ $(0<p<\infty)$ if the subharmonic function $|f|^{p}$ admits a harmonic majorant in D.

Both classes $S(D)$ and $H_{p}(D)$ have local property, i.e., if $f$ is in the class $X(D)$, then $f$ is in $X\left(D^{\prime}\right)$ for any subdomain $D^{\prime} \subset D$. Furtermore, $H_{p} \subset H_{q} \subset S$, for $p \geqq q$.

We state the definition of another class $E_{1}(D)$. Let $D$ be a Jordan domain with the rectifiable boundary and $z=z(w)$ be a one-to-one conformal map of the disc $U:|w|<1$ onto $D$. An analytic function $f(z)$ defined in $D$ is said to belong to the class $E_{1}(D)$ if the function $f(z(w)) z^{\prime}(w)$ is in the class $H_{1}(U)$. It is shown that this definition is independent of the choice of a map $z(w)$ (cf. [6]).

The following lemma will play a fundamental rôle.

Lemma. Let $D_{1}, D_{2}, I, f_{1}, f_{2}$ and $f$ be as in the problem. Assume that the boundaries of $D_{1}$ and $D_{2}$ are rectifiable and $D_{1} \cup I \cup D_{2}$ is a Jordan domain (with the rectifiable boundary). Let $E$ be a subset of I of linear measure zero. For every $\zeta \in I-E$, let $L_{\zeta}^{j}$ be a simple arc in $D_{j}$ terminating at $\zeta(j=1,2)$. Suppose that

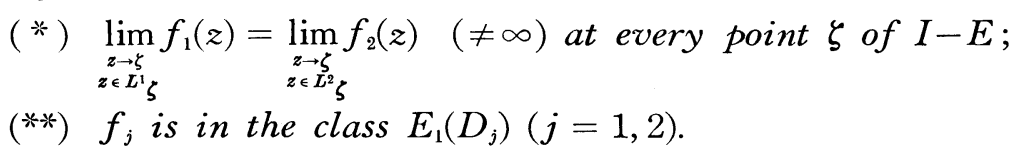

Then $f$ can be extended analytically to the whole $I$ in the sense stated in the problem.

PROOF. By the condition (**) we obtain

$$
\begin{aligned}
\frac{1}{2 \pi i} \int_{\partial D_{j}} \frac{f_{j}^{*}(\zeta)}{\zeta-z} d \zeta & =f_{j}(z) \text { if } \quad z \in D_{j}, \\
& =0 \quad \text { if } \quad z \notin \bar{D}_{j},
\end{aligned}
$$


where $f_{j}^{*}(\zeta)$ is the non-tangential limit of $f_{j}$ at the point $\zeta \in \partial D_{j}$ except for a set of linear measure zero, the integration is taken to the positive sense, and bar means the closure $(j=1,2)$ (cf. chap. 10, $\$ 5$ in [6]). By (*) and by Bagemihl's ambiguous-point theorem [2], we have $f_{1}^{*}(\zeta)=f_{2}^{*}(\zeta)$ except for a set of linear measure zero in $I$. Now we set

$$
F(z)=\frac{1}{2 \pi i} \int_{\partial D} \frac{f^{*}(\zeta)}{\zeta-z} d \zeta, \quad z \in D
$$

where $D=D_{1} \cup I \cup D_{2}$ and $f^{*}(\zeta)=f_{j}^{*}(\zeta), \zeta \in \partial D_{j}(j=1,2)$. Then $F$ is analytic in $D$ and

$$
\begin{aligned}
F(z) & =\frac{1}{2 \pi i} \int_{\partial D_{1}} \frac{f_{1}^{*}(\zeta)}{\zeta-z} d \zeta+\frac{1}{2 \pi i} \int_{\partial D_{2}} \frac{f_{2}^{*}(\zeta)}{\zeta-z} d \zeta \\
& =f_{j}(z) \text { if } \quad z \in D_{j}(j=1,2) .
\end{aligned}
$$

This completes the proof of the lemma.

Now we are ready to prove

THEOREM 1. Let $D_{1}, D_{2}, I, f_{1}, f_{2}$ and $f$ be as in the problem. Assume that $I$ is an open locally rectifiable arc and let $E$ be a subset of I of linear measure zero. For every $\zeta \in I-E$, let $L_{\zeta}^{j}$ be a simple arc in $D_{j}$ terminating at $\zeta(j=1,2)$. Suppose that

(a) $\lim _{\substack{z \rightarrow \zeta \\ z \in L^{1} \zeta}} f_{1}(z)=\lim _{\substack{z \rightarrow \zeta \\ z \in L^{2} \zeta}} f_{2}(z)=\omega_{\zeta}(\neq \infty)$ for every point $\zeta$ of $I-E$;

(b) the function $\varphi(\zeta)=\omega_{\zeta}$ defined on $I-E$ is bounded in some neighbourhood of every point $\zeta$ of $I-E$;

(c) $f_{j}$ is in the class $S\left(D_{j}\right)(j=1,2)$.

Then there exists a closed set e relative to $I$ such that $e$ is a subset of $E$ and $f$ can be extended to be analytic in the open set $I-e$.

PROOF. First we prove :

For any point $\zeta_{0} \in I-E$ there exists a Jordan domain $D_{0}$ with the rectifiable boundary such that

(1) $\xi_{0} \in D_{0}$ and the open set $D_{1} \cup D_{2}$ has no exterior point belonging to $D_{0}$;

(2) $D_{0}^{j}=D_{0} \cap D_{j}$ is a Jordan domain with the rectifiable boundary $(j=1,2)$;

(3) $\bar{D}_{0}^{1} \cap I=\bar{D}_{0}^{2} \cap I=J_{0}$ is a rectifiable arc containing $\zeta_{0}$ in its interior ;

(4) $f$ is bounded in $D_{0}^{1} \cup D_{0}^{2}$. 
By the condition (b) there exists a rectifiable subarc $J_{0}^{1}$ of $I$ such that $J_{0}^{1}$ has $\zeta_{0}$ as its interior point, $I$ has normals from the interior of $D_{1}$ to both terminal points of $J_{0}^{1}$ and such that $\varphi(\zeta)$ is bounded in $J_{0}^{1}-E$. Therefore there exists a Jordan domain $G_{0}^{1} \subset D_{1}$ with the rectifiable boundary such that $\bar{G}_{0}^{1} \cap I=J_{0}^{1}$. Let $z=z(w)$ be a one-to-one comformal map of the unit disc $U$ onto $G_{0}^{1}$. Let $J_{0}^{*}$ be the inverse image of $J_{0}^{1}$ by the natural extension $\zeta=z\left(\zeta^{*}\right)$ of $z=z(w)$ to the unit circle $\left|\zeta^{*}\right|=1$. Set $\zeta_{0}=z\left(\zeta_{0}^{*}\right)$. Evidently the composite function $F_{1}(w)=f_{1}(z(w))$ is in the class $S(U)$ and $F_{1}$ has the asymptotic value $\varphi\left(z\left(\zeta^{*}\right)\right)$ at a.e. (almost every) point $\zeta^{*}$ in the interior $I_{0}^{*}$ of $J_{0}^{*}$. By using Bagemihl's theorem [2] again we know that $F_{1}$ has radial limit $F_{1}\left(\zeta^{*}\right)=\varphi\left(z\left(\zeta^{*}\right)\right)$ at a.e. point $\zeta^{*}$ of $I_{0}^{*}$ since $F_{1}$ is of bounded type. Without loss of generality, we may assume that $\left|F_{1}\left(\zeta^{*}\right)\right|<1$ at a.e. point in $I_{0}^{*}$ since the space $S(U)$ is linear. Let $h(w)$ be the least harmonic majorant of $\log ^{+}\left|F_{1}(w)\right|$ in $U$, which is quasi-bounded, and hence is represented as the Poisson integral of its radial limits. Then $h(w)$ has radial limit $h\left(\zeta^{*}\right)=\log ^{+}\left|F_{1}\left(\zeta^{*}\right)\right|=0$ at a.e. point $\zeta^{*}$ in the open arc $I_{0}^{*}$. Hence $h$ can be continued harmonically across $I_{0}^{*}$. This shows that there exists an open disc $d$ with the centre $\xi_{0}^{*}$ such that $h$ and consequently $F_{1}$ are bounded in $d \cap U$. Therefore there exists an open disc $v_{1}$ with the centre $\zeta_{0}$ such that $f_{1}$ is bounded in $v_{1} \cap D_{1}$. Similarly we can choose a disc $v_{2}$ for $f_{2}$. Now we can make easily $D_{0}^{j} \subset v_{1} \cap D_{j}$ as we wanted $(j=1,2)$.

Next we remark that the derived function $z^{\prime}(w)$ in the definition of the class $E_{1}(D)$ is in the class $H_{1}(U)$ since $D$ has the rectifiable boundary. This shows that any bounded analytic function in $D$ belongs to $E_{1}(D)$. Now we can apply the lemma to $D_{0}^{j}$ and $f_{j}$ since $f_{j} \in E_{1}\left(D_{0}^{j}\right)(j=1,2)$. As a consequence we know that $f$ can be extended analytically to $I_{0}$, the interior of $J_{0}$. Thus we have proved that $f$ can be extended to be analytic to an open arc $I_{\zeta} \subset I$ corresponding to every point $\zeta \in I-E$. Set $e=I-\bigcup_{\zeta \in I-E} I_{\zeta}$. Then $e$ satisfies the conditions of the theorem. This completes the proof of the theorem.

REMARK. Let $A_{\infty}$ be the set of points of $I$ at which at least one of $f_{1}$ and $f_{2}$ has $\infty$ as an asymptotic value. Then, instead of the condition (c) in our theorem, we can take

(c*) $\bar{A}_{\infty} \cap(I-E)=\emptyset$.

In fact, this condition implies that the function $F_{1}(w)=f_{1}(z(w))$ in our proof is bounded in $d \cap U$ since the technique in the proof of Bagemihl-Noshiro's theorem ([3], [9]) is available. The rest of the proof is the same as in ours. Furthermore, this shows that we can mix these two conditions, i.e., we can take the following $\left(c^{* *}\right)$ instead of $(c)$ :

(c**) $f_{1}$ is in the class $S\left(D_{1}\right)$ and ${\overline{A_{\infty}^{2}}}_{\infty} \cap(I-E)=\emptyset$, 
where $A_{\infty}^{2}$ is the set of points in $I$ at which $f_{2}$ has $\infty$ as an asymptotic value.

The problem of finding relations between the conditions (c) and ( $\left.c^{*}\right)$ seems to be open.

3. By a simple open smooth arc we mean a simple open arc $I$ such that at every point $\zeta \in I$ there exists a unique tangent vector $T_{\zeta}$ and such that the angle $\theta(\zeta)$ of the vector $T_{\zeta}$ to the positive real axis is a continuous function of $\zeta \in I$. Clearly a simple open smooth arc is locally rectifiable.

We obtain

TheOREM 2. Let $D_{1}, D_{2}, I, f_{1}, f_{2}$ and $f$ be as in the problem. Assume that $I$ is a simple open smooth arc and let $E$ be a subset of I of linear measure zero. For every $\zeta \in I-E$, let $L_{\zeta}^{j}$ be a simple arc in $D_{j}$ terminating at $\zeta(j=1,2)$. Suppose that

(I) $\lim _{\substack{z \rightarrow \zeta \\ z \in L_{\zeta}}} f_{1}(z)=\lim _{\substack{z \rightarrow \zeta \\ z \in L^{2} \zeta}} f_{2}(z)(\neq \infty)$ for every point $\zeta$ of $I-E$;

(II) $f_{j}$ is in the class $H_{p}\left(D_{j}\right)$ for some $p>1(j=1,2)$.

Then $f$ can be extended analytically to the whole $I$.

Proof. First, by the property of $I$ we can make easily a Jordan domain $D_{0}$ with the rectifiable boundary corresponding to every point $\zeta_{0}$ in the whole $I$ such that the following conditions hold:

(1) the same condition as (1) in the proof of Theorem 1;

(2') $D_{0}^{j}=D_{0} \cap D_{j}$ is a Jordan domain with the smooth boundary $(j=1,2)$;

(3) the same condition as (3) in the proof of Theorem 1.

Here a Jordan curve $J: z=z(t), 0 \leqq t \leqq 1$ is said to be smooth if any simple open subarc of $J$ is smooth and if we denote by $\theta(t)$ the angle of the tangent vector at the point $z(t)(0 \leqq t<1)$ to the real axis, we have $\lim _{t \rightarrow 1} \theta(t)=\theta(0)+2 \pi$. The existence of $D_{0}^{1}$, for example, is shown by the existence of a smooth curve in $D_{1}$ tangent to $I$ at the point near $\zeta_{0}$.

Next we show

(4') $f_{j}$ is in the class $E_{1}\left(D_{0}^{j}\right)(j=1,2)$.

Let $z=z(w)$ be a one-to-one conformal map of the unit disc $U$ onto $D_{0}^{1}$. Then by the well-known theorem (cf. Theorem 5, p. 410, [6]) the function $z^{\prime}(w)$ is in the class $H_{q}(U)$ for any $q>0$. On the other hand, the function $F_{1}(w)$ $=f_{1}(z(w))$ is in the class $H_{p}(U)$. Hence by Hölder's inequality

$$
\int_{0}^{2 \pi}\left|f_{1}\left(z\left(r e^{i \theta}\right)\right) z^{\prime}\left(r e^{i \theta}\right)\right| d \theta \leqq\left(\int_{0}^{2 \pi}\left|F_{1}\left(r e^{i \theta}\right)\right|^{p} d \theta\right)^{1 / p}\left(\int_{0}^{2 \pi}\left|z^{\prime}\left(r e^{i \theta}\right)\right|^{q} d \theta\right)^{1 / q}
$$


with $(1 / p)+(1 / q)=1,0 \leqq r<1$, we know that $f_{1}(z(w)) z^{\prime}(w)$ is in $H_{1}(U)$ and hence $f_{1}$ is in the class $E_{1}\left(D_{0}^{1}\right)$. Similarly $f_{2}$ is in the class $E_{1}\left(D_{0}^{2}\right)$. The rest of the proof is the same as in the proof of Theorem 1 .

4. Under a stronger condition that $I$ is analytic, we obtain

Theorem 3. Let $D_{1}, D_{2}, I, f_{1}, f_{2}$ and $f$ be as in the problem. Assume that $I$ is a simple open analytic arc and let $E$ be a subset of I of linear measure zero. For every $\zeta \in I-E$, let $L_{\zeta}^{j}$ be a simple arc in $D_{j}$ terminating at $\zeta(j=1,2)$. Suppose that

(i) $\lim _{\substack{z \rightarrow \zeta \\ z \in L^{1}}} f_{1}(z)=\lim _{\substack{z \rightarrow \zeta \\ z \in L^{2}}} f_{2}(z)(\neq \infty)$ for every point $\zeta$ of $I-E$;

(ii) $f_{j}$ is in the class $H_{1}\left(D_{j}\right)(j=1,2)$.

Then $f$ can be extended analytically to the whole $I$.

PrOOF. We have only to prove the following:

Let $G$ be the open unit disc $|z|<1, G_{1}$ be the open upper half disc and $G_{2}$ be the open lower half disc. Let $I$ be the open interval $-1<x<1$ on the real axis. Let $g_{j}$ be in $H_{1}\left(G_{j}\right)(j=1,2)$. Assume that

(iii) $\lim _{\substack{z=x \\\left\{z=x \\ z \in G_{1}\right.}} g_{1}(z)=\lim _{\substack { z \rightarrow x \\ \begin{subarray}{c}{z z=x \\ z \in G_{2}{ z \rightarrow x \\ \begin{subarray} { c } { z z = x \\ z \in G _ { 2 } } }\end{subarray}} g_{2}(z)(\neq \infty)$ for a.e. point $x \in I$.

Then there exists an analytic function $g$ in $G$ such that $g=g_{j}$ in $G_{j}(j=1,2)$.

To prove this, set $\psi(z)=g_{1}(z)+\overline{g_{2}}(\bar{z})$ and $\chi(z)=i\left(g_{1}(z)-\overline{g_{2}}(\bar{z})\right)$ for $z \in G_{1}$, where bar means the complex conjugate. Then both $\psi$ and $\chi$ are in $H_{1}\left(G_{1}\right)$ since the function $g^{*}(z)=\overline{g_{2}(\bar{z})}$ for $z \in G_{1}$ is in $H_{1}\left(G_{1}\right)$ and the class $H_{1}\left(G_{1}\right)$ is linear. By the condition (iii) both $\psi$ and $\chi$ have real asymptotic values along the vertical lines at a.e. point in $I$. Let $z=z(w)$ be a one-to-one conformal map of the unit disc $U$ onto $G_{1}$. Then we can apply Rudin's lemma (Lemma 4.4., p. 59, [12]) to the functions $\psi(z(w))$ and $\chi(z(w))$. As a consequence we know that both $\psi$ and $\chi$ can be continued analytically to the whole $G$ and the Schwarz reflexion principle holds. Let $\Psi$ and $\mathrm{X}$ be the resulting functions of $\psi$ and $\chi$ respectively and set $g(z)=\frac{1}{2}(\Psi(z)-i \mathrm{X}(z))$. Then $g(z)=\frac{1}{2}(\psi(z)$ $-i \chi(z))=g_{1}(z)$ in $G_{1}$ and if $\left.z \in G_{2}, g(z)=\frac{1}{2}(\overline{\Psi(\bar{z})}-i \overline{\bar{X}}(\bar{z}))=\frac{1}{2}(\overline{\psi(\bar{z}})-i \overline{\chi(\bar{z})}\right)$ $=g_{2}(z)$. This completes the proof of our assertion.

REMARK. Noshiro [10] remarked that there exists a function $g_{1}(z)$ analytic 
in the upper half plane $\widehat{D_{1}}: \Im^{2}>0$ with the following properties :

(A) $\mathfrak{\jmath} g_{1}(z)>0$ in $\widehat{D}_{1}$;

(B) $g_{1}(z)$ has a real vertical limit at a.e. point on the real axis;

(C) $g_{1}(z)$ has an essential singularity at every point on the real axis.

Now we set $g_{2}(z)=\overline{g_{1}(\bar{z})}$ in the lower half plane $\widehat{D}_{2}$. Then applying SmirnovCargo's Theorem (Theorem 2, [4]) we have $g_{j} \in H_{p}\left(\widehat{D}_{j}\right)$ for any $p, 0<p<1$, since $g_{j}$ takes values in a half plane $(j=1,2)$. The vertical limits of $g_{1}$ and $g_{2}$ coincide at a.e. point on the real axis by the condition (B). Thus we cannot replace the condition (ii) in Theorem 3 by

(ii') $f_{j}$ is in the class $H_{p}\left(D_{j}\right)(j=1,2)$

for $p, 0<p<1$.

5. A totally disconnected compact set $E$ in the plane is said to be null for $H_{p}$ if any element of $H_{p}(\boldsymbol{C E})$ is constant, where $\boldsymbol{C E}$ is the complement of $E$ with respect to the extended plane. It is known that if $E$ is of logarithmic capacity zero, then $E$ is null for any $H_{p}, 0<p<\infty$ ([11], cf. [12], [13] and [15]). As a direct corollary to Theorem 2 (resp. Theorem 3) we have: $A$ compact set of linear measure zero lying on a simple open smooth (resp. analytic) arc is null for $H_{p}, p>1$ (resp. $\left.H_{1}\right)$.

Obviously, any $H_{p}$-null set is an $N_{B}$ set in the sense of Ahlfors and Beurling [1] $(0<p<\infty)$. On the other hand, if $E$, lying on a simple open analytic arc, is an $N_{B}^{*}$ set, then $E$ is of linear measure zero ([1]). This shows that the notion of $H_{p}$-null sets $(p \geqq 1)$ and the notion of $N_{B}$ sets coincide under the restriction of $E$ lying on a simple open analytic arc. We remark also that Havin and Havinson [7] proved: If $E$, lying on a smooth Jordan curve of a special type, is an $N_{B}$ set, then $E$ is of linear measure zero. This shows that the notion of $H_{p}$-null sets $(p>1)$ and the notion of $N_{B}$ sets coincide under their assumption.

It is well known that there exists a compact set of linear measure zero lying on the real axis and of positive logarithmic capacity. This means that Rudin's question $\left(Q_{1}\right)$ (p. $\left.49,[12]\right)$ is answered in the negative for $p \geqq 1$.

\section{REFERENCES}

[1] L. V. Ahlfors AND A. BeuRling, Conformal invariants and function-theoretic null-sets, Acta Math., 83(1950), 101-129.

[2] F. Bagemirl, Curvilinear cluster sets of arbitrary functions, Proc. Nat. Acad. Sci. U.S. A., 41(1955), 379-382.

[ 3 ] F. BAGEMIHL, Analytic continuation and the Schwarz reflection principle, ibid., 51(1964), 378-380.

[4] G. T. CARGo, Some geometric aspects of functions of Hardy class $H^{p}$, Journ. Math. 
Anal. \& Appl., 7(1963), 471-474.

[5] T. Carleman, Théorie de l'intégrale de Fourier, Uppsala, 1944.

[6] G. M. GoluzIN, Geometric theory of functions of a complex variable, 2nd ed., Moscow, 1966, German translation of lst ed., Berlin, 1957.

[ 7 ] V.P. HAVIN AND S. YA. Havinson, Some estimates of analytic capacity, Dokl. Akad. Nauk SSSR, 138(1961), 789-792, in Russian.

[ 8 ] K. E. MEIER, Über die Randwerte meromorpher Funktionen und hinreichende Bedingungen für Regularität von Funktionen einer komplexen Variablen, Comm. Math. Helvet., 24(1950), 238-259.

[9] K. NosHIRO, Some remarks on cluster sets, Journ. d'Anal. Math., 19(1967), 283-294.

[10] K. NosHIRO, Some theorems on cluster sets, Hung-Ching Chow Sixty-fifth Anniversary Volume, Mathematics Research Center, National Taiwan University, Taiwan, December, 1967, 1-6.

[11] M. PARREAU, Sur les moyennes des fonctions harmoniques et analytiques et la classification des surfaces de Riemann, Ann. Inst. Fourier, 3(1952), 103-197.

[12] W. Rudin, Analytic functions of class $H_{p}$, Trans. Amer. Math. Soc., 78(1955), 46-66.

[13] G. C. TUMARKIN AND S. YA. HAVINSON, On removal of singularities of analytic functions of a class (class D), Uspehi Matem. Nauk, 12(1957), 193-199, in Russian.

[14] F. Wolf, Extension of analytic functions, Duke Math. J., 14(1947), 877-887.

[15] S. YAMASHITA, On some families of analytic functions on Riemann surfaces, Nagoya Math. J., 31(1968), 57-68.

MATHEMATICAL INSTITUTE

TÔHOKU UNIVERSITY

SENDAI, JAPAN 
